\title{
Single nucleotide polymorphism rs13042395 in the SLC52A3 gene as a biomarker for regional lymph node metastasis and relapse-free survival of esophageal squamous cell carcinoma patients
}

Hua-Zhen Tan ${ }^{1,2+}$, Zhi-Yong Wu ${ }^{1,3+}$, Jian-Yi Wu ${ }^{1,2}$, Lin Long ${ }^{1,2}$, Ji-Wei Jiao ${ }^{1,2}$, Yu-Hui Peng ${ }^{1,4}$, Yi-Wei Xu ${ }^{1,2,4}$, Shan-Shan Li $i^{1,2}$, Wei Wang ${ }^{1,2}$, Jian-Jun Zhang ${ }^{1,5}$, En-Min Li ${ }^{1,2^{*}}$ and Li-Yan $X_{u^{1,6^{*}}}$

\begin{abstract}
Background: SLC52A3 was recently identified as a susceptibility gene for esophageal squamous cell carcinoma (ESCC). However, associations between the single nucleotide polymorphisms (SNPs) rs 13042395 (C > T) and rs3746803 (G > A) in SLC52A3 and risk, tumor characteristics and survival of ESCC patients remain inconclusive and of unknown prognostic significance.

Methods: Analyses of the association between SNPs in SLC52A3 and ESCC risk were performed on 479 ESCC cases, together with 479 controls, in a case-control study. Blood samples for cases and controls were collected and genotyped by real-time polymerase chain reaction (PCR) using TaqMan assays. Among the 479 ESCC cases, 343 cases with complete clinical data were used to investigate the association between SNPs and ESCC clinical characteristics; 288 cases with complete clinical data and 5-year follow-up data were used to analyze the association between SNPs and prognosis. Dual luciferase reporter assays and electrophoretic mobility shift assays (EMSAs) were used to investigate the biological function of rs13042395.

Results: No association was found between SLC52A3 rs3746803 and susceptibility, tumor characteristics or survival of ESCC patients. For rs13042395, $\Pi$ genotype carriers were likely to have reduced lymph node metastasis (odds ratio $(\mathrm{OR})=0.55,95 \%$ confidence interval $(\mathrm{Cl}), 0.31-0.98)$ and longer relapse-free survival time $(P=0.03)$. Also, both $\mathrm{rs} 13042395$ (hazard ratio $(H R)=0.62,95 \% \mathrm{Cl}, 0.38-0.99)$ and regional lymph node metastasis ( $\mathrm{HR}=2.06,95 \% \mathrm{Cl}, 1.36-3.13$ for $\mathrm{N} 1$ vs. NO; $\mathrm{HR}=2.88,95 \% \mathrm{Cl}, 1.70-4.86$ for $\mathrm{N} 2$ vs. NO; $\mathrm{HR}=2.08$, $95 \% \mathrm{Cl}, 1.01-4.30$ for N3 vs. N0) were independent factors affecting relapse-free survival for ESCC patients who underwent surgery. Dual luciferase reporter assays and EMSAs suggested that the CC genotype of rs13042395 enhanced SLC52A3 expression, probably via binding with specific transcription factors.

(Continued on next page)
\end{abstract}

\footnotetext{
* Correspondence: nmli@stu.edu.cn; lyxu@stu.edu.cn

${ }^{\dagger}$ Equal contributors

${ }^{1}$ Key Laboratory of Molecular Biology in High Cancer Incidence Coastal

Chaoshan Area of Guangdong Higher Education Institutes, Shantou

University Medical College, No. 22, Xinling Road, Shantou 515041, China

Full list of author information is available at the end of the article
} 
(Continued from previous page)

Conclusions: The rs13042395 polymorphism in SLC52A3 is associated with regional lymph node metastasis and relapse-free survival in ESCC patients.

Keywords: Esophageal squamous cell carcinoma, Single nucleotide polymorphism, SLC52A3 gene, Tumor characteristics, Relapse-free survival

\section{Background}

Esophageal cancer (EC) is the tenth most common cancer worldwide [1]. According to a Chinese national annual cancer registration report in 2010, esophageal cancer is the fifth most common malignant tumor in China, with an incidence of $21.88 / 10^{5}$ [2]. EC has two main histologic subtypes: esophageal squamous cell carcinoma (ESCC) and esophageal adenocarcinoma (EAC). ESCC has a distinct geographic distribution worldwide with higher prevalence in central Asia and southern Africa, and accounts for about $90 \%$ of all EC cases in China [3]. The survival for ESCC patients is poor, with a 5-year overall survival rate below $13.0 \%[4,5]$. On one hand, this outcome is partly because of the lack of effective biomarkers for the early detection of ESCC, which results in most ESCC cases presenting at an advanced stage at the time of diagnosis [6]. On the other hand, due to a lack of early warning biomarkers for relapse after surgery, ESCC is difficult to prevent and control relapse and prolong relapse-free survival. Therefore, effective biomarkers for the early detection and relapse of ESCC are urgently needed.

Single nucleotide polymorphisms (SNPs) are regarded as stable and effective biomarkers for prediction of onset and susceptibility, and prognosis of various cancers. In recent years, genome-wide association studies (GWAS) of ESCC in Chinese populations indicate that SNP loci in the PLCE1, CASP8, TMEM173, ATP1B2 and SLC52A3 genes are associated with ESCC susceptibility [7-11]. SLC52A3 (also named C20orf54) on chromosome 20p13 encodes human riboflavin transporter 2 (RFT2), a trans-membrane protein that specifically and efficiently transports riboflavin into cells, playing a role in riboflavin homeostasis $[12,13]$. More importantly, it has been reported that SLC52A3 is frequently overexpressed in tumors, compared with normal adjacent tissue, in ESCC patients. Knockdown of SLC52A3 in ESCC cells results in inhibition of cell proliferation, colony formation and anchorage-independent growth, whereas overexpression of SLC52A3 in ESCC cells promotes cell proliferation, confers resistance to cisplatin and enhances tumorigenicity in nude mice [14]. All these indicate that SLC52A3 plays an important role in ESCC tumorigenesis and prognosis. A recent GWAS study and smaller studies have shown that some SNP loci, such as rs13042395 $(\mathrm{C}>\mathrm{T}), \mathrm{rs} 3746802(\mathrm{~T}>\mathrm{C}), \operatorname{rs3746803}(\mathrm{G}>\mathrm{A})$ and rs3746804 $(\mathrm{G}>\mathrm{A})$ in SLC52A3, are associated with ESCC risk [8, 15, 16]. The rs13042395 is a SNP locus, located at the $5^{\prime}$ flanking region of the SLC52A3 gene, with a minor allele frequency (MAF) ranging from 9.30 to $36.4 \%$ in ESCC vs. $8.28 \%$ to $36.5 \%$ in controls [8, 9, 1720]. However, according to other GWAS studies and smaller sample replication studies, associations between rs13042395 and ESCC are inconclusive [9, 11, 17-20]. The rs3746803, located in the coding region of SLC52A3, is a functional polymorphism (missense) and site of modification by protein kinase $\mathrm{C}$. The reported MAF of rs3746803 in the PubMed SNP database is $9.05 \%$. So far, only one study demonstrates that no relationship exists between rs3746803 and risk of ESCC [15]. Moreover, neither rs13042395 nor rs3746803 have been validated in regard to whether they are related to ESCC in the Chaoshan area of China, a coastal high-risk area for EC, and it has yet to be reported whether SNPs rs13042395 and rs3746803 are associated with tumor characteristics and survival in ESCC patients.

In the present study, we investigated the association between rs13042395 or rs3746803, in SLC52A3, and ESCC risk, tumor characteristics and survival. We used a large Chinese study population (in the Chaoshan area) that has detailed clinical data and a follow-up time of 5 years. SNPs were genotyped by the Taqman polymerase chain reaction (PCR) method, and luciferase reporter assays and electrophoretic mobility shift assays (EMSAs) were conducted to explore how the SNPs regulate SLC52A3 expression.

\section{Methods \\ Study population}

Study participants for the present study were drawn from the Chaoshan region in China (a coastal high-risk area for ESCC). Analyses of the association between SLC52A3 SNPs and ESCC risk were performed on 479 ESCC cases together with 479 controls. All ESCC cases were diagnosed histopathologically. The controls were matched by gender and age, and were selected from healthy persons who had physical examinations in Shantou Central Hospital (cancer patients were excluded). Blood samples for cases and controls were collected between January 2008 and January 2014. The volume of blood samples of all ESCC cases and controls was more than 3 milliliters. Three hundred forty-three, of the 479 ESCC cases, were used to analyze association between SNPs and ESCC tumor characteristics because they had undergone surgery 
and had detailed clinical data (Table 1). Clinical data for ESCC cases was retrieved from Shantou Central Hospital and the Cancer Hospital of Shantou University Medical College. Two hundred eighty-eight of the 343 cases participated in follow-up studies performed from the 1st of January, 2008, until the 31st of December 2014. Information about the date of death and relapse after surgery was collected. Detailed clinical data and follow-up data of the 288 ESCC cases were used to analyze the association between SNPs and survival of ESCC patients. All participants in the present study have signed informed consent. This study was approved by the Ethics Committee of Shantou University Medical College.

\section{DNA extraction and SNP genotyping}

Genomic DNA was extracted from whole blood with a TIANamp Blood DNA Kit (TIANGEN BIOTECH, Beijing, China). Genotyping was performed using a TaqMan PCR allelic discrimination method with an ABI 7500 Real-Time PCR System (Applied Biosystems, Foster City, California, USA). Predesigned TaqMan SNP genotyping assays were used, with minor groove binding probes 5 '-labelled with VIC or FAM fluorophores (Applied Biosystems). PCR was performed with $20 \mathrm{ng}$ genomic DNA in a total reaction volume of $5 \mu \mathrm{l}$, using 40x Taqman SNP Genotyping Assay (Applied Biosystems), $2 \times$ Taqman Genotyping Master Mix (Applied Biosystems) and water. PCR was performed under the following conditions: an initial holding at $95{ }^{\circ} \mathrm{C}$ for $10 \mathrm{~min}, 40$ cycles of denaturation at $92{ }^{\circ} \mathrm{C}$ for $15 \mathrm{~s}$ and annealing and extension at $60{ }^{\circ} \mathrm{C}$ for $1 \mathrm{~min}$, and a final holding at $60{ }^{\circ} \mathrm{C}$ for $1 \mathrm{~min}$. All blood samples were genotyped successfully.

\section{Assembly of reporter constructs}

We prepared a 400 bp genomic DNA fragment containing the human rs13042395 locus located 5622 nt upstream of the transcriptional starting site in the human SLC52A3 gene. The fragment was generated by PCR with a forward primer containing a $K p n \mathrm{I}$ site (underlined) 5' -GGTACCTAATGCGTGGGCGACAGA-3' and a reverse primer containing an XhoI site (underlined) 5' CTCGAGGTGGCAAGCCAGATGGT-3' and inserted into the pGL3-Promoter (pGLP) reporter vector (Promega, Madison, WI, USA) to create the pGLP-C construct, in which the SNP locus contained the $C$ allele. The pGLP-C construct then underwent site-directed mutagenesis to engineer the pGLP-T construct in which the SNP locus contained the $\mathrm{T}$ allele. Mutagenesis was conducted by PCR (primers: 5'-CAGGGCCAGTGCACCGTTATTG TGTGGGCTGGG-3'; 5'-AACGGTGCACTGGCCCTG GTCAGAACCCCACTC-3') using the Fast Mutagenesis System (TransGen Biotech, Beijing, China).

\section{Cell culture and dual luciferase reporter assay}

The human KYSE150 and KYSE180 esophageal squamous carcinoma cell lines were cultured in RPMI-1640 medium (ThermoFisher, HyClone, CA, USA) supplemented with $10 \%$ fetal bovine serum (Life Technologies, Australia). All cells were maintained at $37{ }^{\circ} \mathrm{C}$ in a humidified $5 \% \mathrm{CO}_{2}$ atmosphere.

Cells were inoculated in 96-well plates at $1.5 \times 10^{5}$ cells/ $\mathrm{ml}$, grown to $50-80 \%$ confluence and co-transfected with $0.5 \mu \mathrm{g}$ of either pGLP-C or pGLP-T, and $0.01 \mu \mathrm{g}$ control vector (Renilla luciferase plasmid pRL-TK (Promega, Madison, WI, USA)), using Superfect Transfection Reagent (QIAGEN, Hilden, Germany) according to the manufacturer's instructions. The experimental reporter vector contained a modified coding region for firefly (Photinuspyralis) luciferase that has been optimized for monitoring transcriptional activity in transfected eukaryotic cells. After transfection, cells were incubated for $48 \mathrm{~h}$ and harvested in Passive Lysis Buffer (Promega, Madison, WI, USA). The luciferase reporter activity of the lysates was measured using the Dual-Luciferase Reporter Assay System (Promega, Madison, WI, USA) according to the manufacturer's recommendations.

\section{Electrophoretic mobility shift assay (EMSA)}

Nuclear extracts from KYSE150 cells were prepared using NE-PER Nuclear and Cytoplasmic Extraction Reagent Kit (Thermo Pierce Biotechnology, Rockford, IL, USA) according to the manufacturer's instructions. EMSA was performed using biotin 3 '-end labelled $30 \mathrm{bp}$ probes for the rs13042395 locus. Equimolar amounts of complementary and single-stranded oligonucleotides were annealed. The oligonucleotide probes used in EMSAs were 5' - GGC CAGTGCACCGTCEATTGTGTGGGCTGGG-3' and 5'CCCAGCCCACACAATGACGGTGCACTGGCC-3' for the CC genotype; and $\overline{5}^{\prime}$-GGCCAGTGCACCGTTATT GTGTGGGCTGGG-3' and 5' -CCCAGCCCACACA-ATA ACGGTGCACTGGCC-3' for 'TT genotype, in which underlined nucleotides indicate the SNP locus. In specific competition experiments, a 200-fold molar excess of unlabeled oligonucleotides was added to the binding reaction. Probes were incubated with $3 \mu \mathrm{g}$ of nuclear protein extracts for $25 \mathrm{~min}$ at room temperature. The remaining steps followed the Light Shift Chemiluminescent EMSA Kit protocol (Thermo Pierce Biotechnology, Rockford, IL, USA).

\section{Statistical analysis}

The observed genotype frequencies in the controls were tested for Hardy-Weinberg equilibrium using free online software (http://analysis.bio-x.cn/myAnalysis.php) [21]. Odds ratios (ORs) and $95 \%$ confidence intervals (95\% CIs) for association between the SLC52A3 SNPs and ESCC risk were calculated by binary logistic regression. 
Table 1 Characteristics of ESCC cases and controls

\begin{tabular}{|c|c|c|c|c|}
\hline \multirow[t]{2}{*}{ Variables } & \multicolumn{2}{|c|}{ SNP and ESCC risk } & \multirow{2}{*}{$\begin{array}{l}\text { SNP and ESCC } \\
\text { progression } \\
\text { ESCC } \\
(N=343)\end{array}$} & \multirow{2}{*}{$\begin{array}{l}\text { SNP and ESCC } \\
\text { prognosis } \\
\text { ESCC } \\
(N=288)\end{array}$} \\
\hline & $\begin{array}{l}\text { ESCC } \\
(N=479)\end{array}$ & $\begin{array}{l}\text { Control } \\
(N=479)\end{array}$ & & \\
\hline \multicolumn{5}{|l|}{ Gender } \\
\hline Female & 109 & 109 & 87 & 79 \\
\hline Male & 370 & 370 & 256 & 209 \\
\hline $\begin{array}{l}\text { Age (years) } \\
(\mathrm{M} \pm \mathrm{SD})\end{array}$ & $59.92 \pm 9.34$ & $59.61 \pm 8.84$ & $58.39 \pm 8.83$ & $58.28 \pm 8.84$ \\
\hline \multicolumn{5}{|c|}{ Size of tumor $(\mathrm{cm})$} \\
\hline$\leq 3$ & 101 & & 98 & 89 \\
\hline $4-5$ & 160 & & 158 & 130 \\
\hline$\geq 6$ & 89 & & 87 & 69 \\
\hline Unknown & 129 & & 0 & 0 \\
\hline \multicolumn{5}{|c|}{$\begin{array}{l}\text { Depth of tumor } \\
\text { invasion }\end{array}$} \\
\hline Tis & 8 & & 8 & 5 \\
\hline $\mathrm{T} 1$ & 29 & & 29 & 24 \\
\hline $\mathrm{T} 2$ & 45 & & 45 & 39 \\
\hline T3 & 239 & & 239 & 216 \\
\hline T4 & 22 & & 22 & 4 \\
\hline Unknown & 136 & & 0 & 0 \\
\hline \multicolumn{5}{|c|}{$\begin{array}{l}\text { Regional lymph } \\
\text { node metastasis }\end{array}$} \\
\hline NO & 178 & & 178 & 150 \\
\hline $\mathrm{N} 1$ & 103 & & 103 & 88 \\
\hline N2 & 40 & & 40 & 34 \\
\hline N3 & 22 & & 22 & 16 \\
\hline Unknown & 136 & & 0 & 0 \\
\hline \multicolumn{5}{|c|}{ Distant metastasis } \\
\hline MO & 337 & & 337 & 288 \\
\hline M1 & 6 & & 6 & 0 \\
\hline Unknown & 136 & & 0 & 0 \\
\hline \multicolumn{5}{|c|}{ Tumor location } \\
\hline $\begin{array}{l}\text { Upper } \\
\text { thoracic }\end{array}$ & 56 & & 27 & 19 \\
\hline $\begin{array}{l}\text { Middle } \\
\text { thoracic }\end{array}$ & 251 & & 191 & 158 \\
\hline $\begin{array}{l}\text { Lower } \\
\text { thoracic }\end{array}$ & 143 & & 125 & 111 \\
\hline Unknown & 29 & & 0 & 0 \\
\hline \multicolumn{5}{|c|}{ TNM classification } \\
\hline 0 & 8 & & 8 & 4 \\
\hline I & $42(3)^{a}$ & & 39 & 35 \\
\hline$\|$ & $156(18)^{a}$ & & 138 & 124 \\
\hline III & $185(33)^{a}$ & & 152 & 125 \\
\hline IV & $20(14)^{a}$ & & 6 & 0 \\
\hline Unknown & 68 & & 0 & 0 \\
\hline
\end{tabular}

Table 1 Characteristics of ESCC cases and controls (Continued)

\begin{tabular}{lll}
\hline Radiotherapy after surgery & 204 & 204 \\
No & 84 & 84 \\
Yes & 55 & 0 \\
Unknown & & \\
Chemotherapy after surgery & 207 & 207 \\
No & 81 & 81 \\
Yes & 55 & 0 \\
Unknown & &
\end{tabular}

All associations are significant at $P<0.05$

ESCC esophageal squamous cell carcinoma, SNP single nucleotide polymorphism, $M$ mean, $S D$ standard deviation

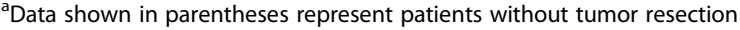

Ordinal logistic regression was performed to estimate OR and $95 \% \mathrm{CI}$ for association between SNPs and ESCC characteristics. Survival curves for ESCC relapse-free survival and overall survival after surgery were derived by the Kaplan-Meier method. Univariate Cox regression was used to estimate hazard ratio (HR) and $95 \% \mathrm{CI}$ for risk factors related to ESCC relapse-free survival and overall survival, respectively. Furthermore, in order to determine the value of certain risk factors as independent prognostic factors, multivariate Cox regression was performed to analyze the HR of the overall risk factors (adjusted for each other) for ESCC relapse-free survival and overall survival. TMN stage was composed of depth of tumor invasion $(\mathrm{T})$, regional lymph node metastasis $(\mathrm{N})$ and distant metastasis $(\mathrm{M})$. There were collinearities between TNM stage and tumor invasion, regional lymph node metastasis and distant metastasis, respectively. In order to get more information about the relation between tumor invasion, regional lymph node metastasis, distant metastasis and survival, we excluded TNM stage, in the multivariate Cox analysis, by a forward stepwise method. Relative luciferase activity was defined as firefly luciferase activity per Renilla luciferase activity in transfected cells. One-way analysis of variance along with the Bonferroni post hoc test was used to determine whether differences were significant for relative luciferase activity between groups. All analyses mentioned above were performed using SPSS, version 16.0 software (IBM SPSS, Chicago, IL, USA). All $P$-values were 2 -sided, and a value of less than 0.05 was considered as having statistical significance.

\section{Results}

\section{Lack of association between SLC52A3 SNPs and ESCC} susceptibility

The observed genotype frequencies for the two polymorphisms of SLC52A3 in the controls conformed to the Hardy-Weinberg equilibrium $(P=0.06$ and 0.80 for rs13042395 and rs3746803, respectively). No significant 
associations were observed between rs13042395 or SNP 3746803 and ESCC risk $(P>0.05$, Table 2$)$.

\section{Association of SNPs with ESCC tumor characteristics at the time of diagnosis}

Significant associations were observed when we stratified the ESCC cases by tumor characteristics (Table 3). For rs13042395, TT genotype carriers were less likely to have regional lymph node metastasis $(\mathrm{OR}=0.55,95 \% \mathrm{CI}$, 0.31-0.98) than CC genotype carriers. This meant the risk of having a higher degree of regional lymph node metastasis for TT genotype carriers was 0.55 -fold less than that for CC genotype carriers in ESCC patients. No association was found between rs13042395 and other tumor characteristics such as tumor size, depth of tumor invasion, distant metastasis and TNM classification. For rs3746803, no associations were observed with ESCC tumor characteristics.

\section{Univariate and multivariate analysis of SNPs with ESCC relapse-free survival}

For rs13042395, the median relapse-free survival time for $(\mathrm{CC}+\mathrm{CT})$ genotype carriers and TT genotype carriers was 23 and 30 months, respectively. Relapse-free survival time of TT genotype carriers was significantly longer compared with that of $(\mathrm{CC}+\mathrm{CT})$ genotype carriers $(P=$ 0.03) (Fig. 1b). According to univariate Cox regression analysis, TT genotype carriers had a decreased risk for relapse after surgery $(\mathrm{HR}=0.60,95 \% \mathrm{CI}, 0.38-0.96)$ compared with $(\mathrm{CC}+\mathrm{CT})$ genotype carriers (Table 4).

Table 2 Association between SNPs in SLC52A3 and ESCC risk in the Chinese population $\left(\mathrm{N}_{\mathrm{ESCC}}=479, \mathrm{~N}_{\text {control }}=479\right)$

\begin{tabular}{cllll}
\hline Genotype & ESCC & Control & OR (95 \% Cl) & $P$ \\
& $N(\%)$ & $N(\%)$ & & \\
\hline rs13042395 & & & & \\
CC & $135(28.18)$ & $147(30.69)$ & 1.00 (reference) & \\
CT & $236(49.27)$ & $218(45.51)$ & $1.18(0.88-1.59)$ & 0.28 \\
TT & $108(22.55)$ & $114(23.80)$ & $1.03(0.73-1.47)$ & 0.86 \\
CT TT & $344(71.82)$ & $332(69.31)$ & $1.14(0.86-1.50)$ & 0.36 \\
CC + CT & $371(77.45)$ & $365(76.20)$ & 1.00 (reference) & \\
TT & $108(22.55)$ & $114(23.80)$ & $0.93(0.69-1.26)$ & 0.65 \\
rs3746803 & & & & \\
GG & $425(88.73)$ & $424(88.52)$ & 1.00 (reference) & \\
GA & $55(11.48)$ & $53(11.06)$ & $1.00(0.67-1.49)$ & 0.99 \\
AA & $1(0.21)$ & $2(0.42)$ & $0.50(0.05-5.52)$ & 0.57 \\
GA + AA & $54(11.27)$ & $55(11.48)$ & $0.98(0.66-1.46)$ & 0.92 \\
GG + GA & $478(99.79)$ & $477(99.58)$ & 1.00 (reference) & \\
AA & $1(0.21)$ & $2(0.42)$ & $0.50(0.05-5.52)$ & 0.57 \\
\hline
\end{tabular}

All associations are significant at $P<0.05$

SNP single nucleotide polymorphism, ESCC esophageal squamous cell carcinoma, OR odds ratio, $95 \%$ Cl $95 \%$ confidence interval
This result suggests that the relative risk of relapse after surgery for TT genotype carriers is 0.60 -fold less than that for the $(\mathrm{CC}+\mathrm{CT})$ genotype carriers in ESCC patients. Also, depth of tumor invasion, regional lymph node metastasis and TNM classification were risk factors for ESCC relapse-free survival with the $P$-value for trend $\left(P_{\text {trend }}\right)<0.05$. In multivariate Cox regression models, gender, age, rs13042395, rs3746803, tumor size, depth of tumor invasion, regional lymph node metastasis, tumor location, TNM classification, radiotherapy after surgery and chemotherapy after surgery were adjusted for each other. Multivariate analysis showed that rs13042395 (HR = 0.62, $95 \% \mathrm{CI}, 0.38-0.99$ for TT vs. $(\mathrm{CC}+\mathrm{CT}))$, depth of tumor invasion, regional lymph node metastasis $(\mathrm{HR}=$ 2.06, $95 \%$ CI, 1.36-3.13 for N1 vs. N0; $\mathrm{HR}=2.88,95 \% \mathrm{CI}$, $1.70-4.86$ for $\mathrm{N} 2 v s$. N0; HR = 2.08, $95 \% \mathrm{CI}, 1.01-4.30$ for $\mathrm{N} 3$ vs. N0) and radiotherapy after surgery $(\mathrm{HR}=1.68,95 \%$ CI, 1.16-2.43) were linked with ESCC relapse (Table 4). Cox regression analysis in 84 patients who had radiotherapy after surgery showed that TT genotype carriers were more likely to have relapse-free survival $(\mathrm{HR}=0.46$, $95 \%$ CI, 0.21-0.98) compared with $(\mathrm{CC}+\mathrm{CT})$ genotype carriers (Additional file 1: Table S1). This indicates that the four factors were independent prognostic factors for ESCC relapse. However, rs3746803 was not shown to be associated with ESCC relapse-free survival either by univariate or multivariate analysis (Table 4, Additional file 2: Figure S1A).

\section{Univariate and multivariate analysis of SNPs with ESCC overall survival}

According to Kaplan-Meier survival curve, the differences of overall survival among genotypes of rs13042395 were not significant (Fig. 1d-f). Also, the difference of overall survival between GG and (GA + AA) genotype of rs3746803 was not significant (Additional file 2: Figure $\mathrm{S} 1 \mathrm{~B})$. Univariate Cox regression analysis showed that neither rs13042395 nor rs3746803 was associated with ESCC overall survival after surgery (Additional file 3: Table S2). In multivariate Cox regression analysis, significant association with ESCC overall survival was found for regional lymph node metastasis, TNM classification and radiotherapy after surgery, but not for rs13042395 or rs3746803 (Additional file 3: Table S2).

\section{Transcriptional activity of the rs13042395 in ESCC cells}

A dual luciferase reporter assay was conducted to investigate the transcriptional activity of rs13042395 in KYSE150 and KYSE180 cells (Fig. 2a). Empty vector pGLP-V was used as a control. The assay showed that both pGLP-C and pGLP-T had greater relative luciferase activity than the control vector when expressed in KYSE150 and KYSE180 cells. The increased range for pGLP-C relative luciferase activity was greatly elevated $(P<0.01)$, while 
Table 3 Association between SNPs in SLC52A3 and clinical characteristics of ESCC in the Chinese population $\left(N_{E S C C}=343\right)$

\begin{tabular}{|c|c|c|c|c|c|c|c|c|c|c|}
\hline \multirow[t]{2}{*}{ Genotype } & \multicolumn{2}{|c|}{$\begin{array}{l}\text { Tumor size } \\
(\leq 3 \mathrm{~cm} / 4-5 \mathrm{~cm} / \geq 6 \mathrm{~cm})\end{array}$} & \multicolumn{2}{|c|}{$\begin{array}{l}\text { Depth of tumor invasion } \\
((\mathrm{Tis}+\mathrm{T} 1) / \mathrm{T} 2 / \mathrm{T} 3 / \mathrm{T} 4)\end{array}$} & \multicolumn{2}{|c|}{$\begin{array}{l}\text { Regional lymph node } \\
\text { metastasis (N0/N1/N2/N3) }\end{array}$} & \multicolumn{2}{|l|}{$\begin{array}{l}\text { Distant metastasis } \\
\text { (M0/M1) }\end{array}$} & \multicolumn{2}{|l|}{$\begin{array}{l}\text { TNM classification } \\
((0+\mathrm{I}) / \mathrm{II} / \mathrm{II} / \mathrm{IV})\end{array}$} \\
\hline & OR $(95 \% \mathrm{Cl})$ & $P$ & OR (95 \% Cl) & $P$ & OR $(95 \%$ Cl) & $P$ & OR $(95 \% \mathrm{Cl})$ & $P$ & OR $(95 \%$ Cl) & $P$ \\
\hline \multicolumn{11}{|l|}{ rs13042395 } \\
\hline $\mathrm{CC}$ & 1.00 (Reference) & & 1.00 (Reference) & & 1.00 (Reference) & & 1.00 (Reference) & & 1.00 (Reference) & \\
\hline$C T$ & $1.17(0.73-1.86)$ & 0.53 & $0.90(0.52-1.55)$ & 0.71 & $0.83(0.52-1.33)$ & 0.45 & $0.83(0.14-5.04)$ & 0.84 & $0.79(0.41-1.26)$ & 0.33 \\
\hline$\pi$ & $1.18(0.67-2.06)$ & 0.57 & $0.82(0.43-1.56)$ & 0.55 & $0.55(0.31-0.98)$ & 0.04 & $0.59(0.05-6.63)$ & 0.67 & $0.72(0.49-1.27)$ & 0.25 \\
\hline$C T+T$ & $1.17(0.75-1.82)$ & 0.49 & $0.88(0.52-1.46)$ & 0.61 & $0.73(0.47-1.14)$ & 0.16 & $0.75(0.14-4.17)$ & 0.74 & $0.76(0.49-1.20)$ & 0.24 \\
\hline$C C+C T$ & 1.00 (Reference) & & 1.00 (Reference) & & 1.00 (Reference) & & 1.00 (Reference) & & 1.00 (Reference) & \\
\hline$\pi$ & $1.07(0.67-1.70)$ & 0.79 & $0.88(0.52-1.50)$ & 0.63 & $0.62(0.38-1.01)$ & 0.06 & $0.66(0.08-5.77)$ & 0.71 & $0.84(0.52-1.34)$ & 0.46 \\
\hline \multicolumn{11}{|l|}{ rs3746803 } \\
\hline GG & 1.00 (Reference) & & 1.00 (Reference) & & 1.00 (Reference) & & 1.00 (Reference) & & 1.00 (Reference) & \\
\hline $\mathrm{GA}+\mathrm{AA}$ & $1.1(0.57-2.09)$ & 0.78 & $1.4(0.65-3.00)$ & 0.39 & $1.06(0.55-2.03)$ & 0.87 & $1.73(0.20-15.02)$ & 0.62 & $1.3(0.67-2.51)$ & 0.43 \\
\hline
\end{tabular}

All associations are significant at $P<0.05$

SNP single nucleotide polymorphism, ESCC esophageal squamous cell carcinoma, OR odds ratio, $95 \% \mathrm{Cl} 95 \%$ confidence interval

that for the pGLP-T was not obvious. This suggests that the CC genotype of rs13042395 has stronger transcription activity for the down-stream gene (SLC52A3) and promotes SLC52A3 expression.

\section{DNA-binding activity for CC genotype of rs13042395}

In order to identify whether DNA-binding activity on rs13042395 plays an important role in SLC52A3 overexpression, we used an EMSA assay to search for potential factors that could interact with rs13042395 (Fig. 2b). Nuclear extracts prepared from KYSE150 cells were incubated with biotin-labeled oligonucleotides for rs13042395 loci containing either the CC or TT genotype. This binding reaction generated four protein-DNA complexes (I, II, III and IV) between the CC genotype oligonucleotides and nuclear protein (shifted bands) (Fig. 2b, lane 2). To determine the specificity of the binding complex, we added a 200-fold molar excess of unlabeled oligonucleotide to the reaction. As a result, the shifted bands were completely ablated (Fig. 2b, lanes 3 and 8). Interestingly, neither protein-DNA complex II nor complex III was generated with the TT genotype oligonucleotide. Collectively, these results demonstrate that a specific interaction exists between nuclear proteins and the DNA sequence containing $\mathrm{C}$ allele rather than the $\mathrm{T}$ allele in the rs13042395 locus. These results are consistent with the above results demonstrating that the CC genotype of rs13042395 in humans promotes $S L C 52 A 3$ gene expression, probably via binding with additional transcription factors.

\section{Discussion}

In recent years, the role of rs13042395 in ESCC susceptibility has been controversial. In 2010, rs13042395 was initially found to be related to ESCC by Wang et al. [8]. However, in later years, researchers found no association between this SNP and ESCC [17-20]. Our results are consistent with the latter. The present study shows that neither rs13042395 nor rs3746803 is related to ESCC risk. For rs13042395, TT genotype carriers were less likely to have regional lymph node metastasis (compared with CC genotype carriers) and more likely to have better relapse-free survival (compared with $(\mathrm{CC}+\mathrm{CT})$ genotype carriers). The CC genotype of rs13042395 likely promotes down-stream SLC52A3 gene expression in human ESCC, probably by binding with specific transcription factors.

According to our study, rs13042395 in SLC52A3 plays an important role in regional lymph node metastasis. This is supported by our demonstration that rs13042395 and regional lymph node metastasis status are independent prognostic factors for relapse-free survival. Moreover, we show that the CC genotype of rs13042395 could act by promoting SLC52A3 expression by causing the binding of additional transcription factors. Previous studies demonstrated that regional lymph node metastasis is significantly associated with relapse-free survival and overall survival in ESCC patients [22, 23]. More lymph node metastasis leads to worse survival. Also, it has been demonstrated that the SLC52A3 gene is overexpressed in ESCC cells, promotes ESCC cell proliferation and protects against cell death [14]. Increases in cancer cell proliferation and decreases in cancer cell death will inevitably promote cancer cell metastasis to lymph nodes and thereby result in poor survival in ESCC patients. Therefore, it is reasonable to conclude that the CC genotype of rs13042395 in humans leads to SLC52A3 overexpression, which promotes ESCC cell proliferation and protects against cell death. This biologic function makes CC genotype carriers more likely to have lymph node metastasis and worse relapse-free survival. Conversely, TT genotype carriers are less likely to have lymph node metastasis and better relapse-free survival, as observed in present study. All evidence indicates that rs13042395 plays 

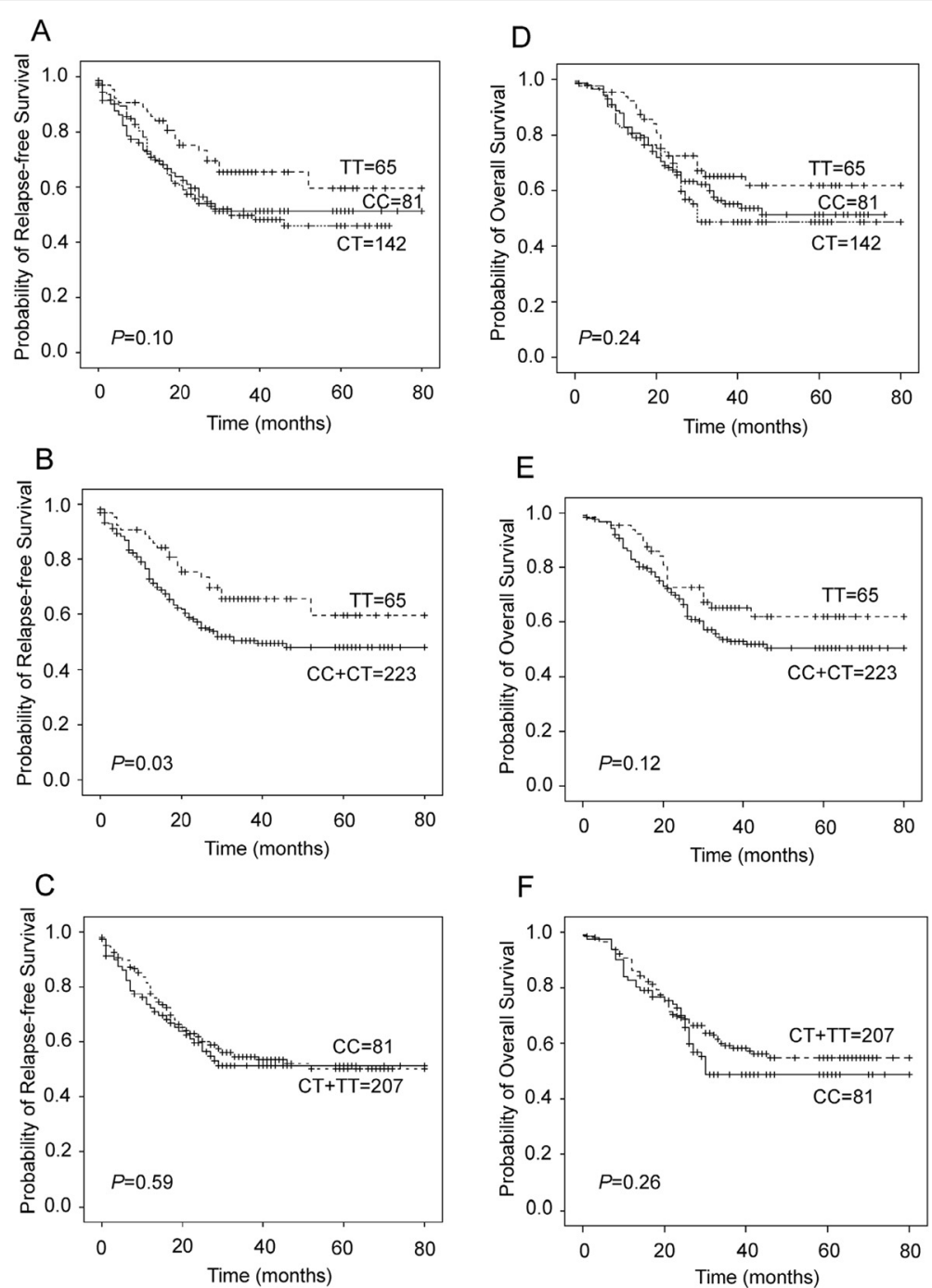

Fig. 1 Kaplan-Meier analysis of relapse-free survival and overall survival for rs13042395 polymorphisms in the SLC52A3 gene in ESCC cases. a The median relapse-free survival time for CC, CT and $\Pi$ genotype carriers was 24,23 and 30 months, respectively. The differences of relapse-free survival time among $C C, C T$ and $\Pi$ genotype carriers were not significant $(P=0.10)$. $\mathbf{b}$ The median relapse-free survival time for $(C C+C T)$ genotype carriers and $T$ genotype carriers was 23 and 30 months, respectively. Relapse-free survival time of $T$ genotype carriers was significantly longer compared with that of $(C C+C T)$ genotype carriers $(P=0.03)$. $\mathbf{c}$ The median relapse-free survival time for $C C$ genotype carriers and $(C T+T T)$ genotype carriers was 24 and 25 months, respectively. The difference of relapse-free survival time between $C C$ and $(C T+T T)$ genotype carriers was not significant $(P=0.59)$. $\mathbf{d}$ The median overall survival time for $C C, C T$ and $T$ genotype carriers was 26,27 and 32 months, respectively. The differences of overall survival time among CC, CT and TT genotype carriers were not significant $(P=0.24)$. e The median overall survival time for $(C C+C T)$ genotype carriers and $T T$ genotype carriers was 27 and 32 months, respectively. The difference of overall survival time between $(C C+C T)$ and $T$ genotype carriers was not significant $(P=0.12)$. $\mathbf{f}$ The median overall survival time for $C C$ genotype carriers and $(C T+T T)$ genotype carriers was 26 and 29 months, respectively. The difference of overall survival time between $C C$ and $(C T+T T)$ genotype carriers was not significant $(P=0.26)$

an essential role in ESCC prognosis and is a potential predictive marker for progression and prognosis of ESCC.

RFT2 is a crucial transporter, encoded by the $S L C 52 A 3$ gene, involved in epithelial cell uptake of riboflavin for nutritional utilization [12, 13]. Riboflavin deficiency has been identified as a risk factor for ESCC in high-risk areas [24-27]. Prior research indicates that dietary riboflavin could ameliorate the effects of ESCC carcinogens
[28]. Large population intervention trials also suggest that riboflavin supplementation can reduce the incidence of esophageal cancer [29-32]. However, in the ESCC patients supplemented with riboflavin, blood riboflavin in $34.2 \%$ of the subjects was still lower than normal, despite sufficient dietary riboflavin [32]. Our study shows that the CC genotype of rs13042395 can promote SLC52A3 expression. A recent study indicates that, in ESCC, high 
Table 4 Univariate analyses and multivariate analysis of factors associated with relapse-free survival for ESCC patients

\begin{tabular}{llll}
$\left(\mathrm{N}_{\mathrm{ESCC}}=288\right)$ & \\
\hline Variables & $\mathrm{HR}$ & $\frac{95 \% \mathrm{Cl} \text { for HR }}{\text { Lower Upper }}$
\end{tabular}

Univariate analyses

Gender

$\begin{array}{lllll}\text { Female } & 1.00 & \text { Reference } & & \\ \text { Male } & 1.12 & 0.75 & 1.66 & 0.58 \\ \text { (years) } & 0.99 & 0.97 & 1.01 & 0.42\end{array}$

Age (years)

(1)

CC

CT

$\Pi$

$\mathrm{CT}+\mathrm{TT}$

$$
1.00
$$

1.05

0.62

0.90

$\mathrm{CC}+\mathrm{CT}$

1.00

$\pi$

rs3746803

$$
\mathrm{GG}
$$

1.00

1.23

Tumor size $(\mathrm{cm})$

$\begin{array}{ll}\leq 3 & 1.00 \\ 4-5 & 1.48 \\ \geq 6 & 1.40 \\ P_{\text {trend }} & \end{array}$

Depth of tumor invasion

Tis $+\mathrm{T}$
$\mathrm{T} 2$
$\mathrm{~T} 3$
$\mathrm{~T} 4$
$P_{\text {trend }}$

Regional lymph node metastasis

$\begin{array}{ll}\text { No } & 1.00 \\ \text { N1 } & 2.63 \\ \text { N2 } & 3.73 \\ \text { N3 } & 3.46 \\ P_{\text {trend }} & \end{array}$

Reference

1.74

3.98

2.24

6.23

6.91

Tumor location

Upper thoracic
Middle thoracic
Lower thoracic
$P_{\text {trend }}$

1.00
0.63
0.72

Reference

0.34

1.16

0.14

0.31

0.31

TNM classification

$\begin{array}{lllll}0+1 & 1.00 & \text { Reference } & \\ \| & 5.83 & 1.81 & 18.76 & 0.003 \\ \text { III } & 14.21 & 4.47 & 45.17 & <0.001 \\ P_{\text {trend }} & & & & <0.001\end{array}$

Table 4 Univariate analyses and multivariate analysis of factors

\begin{tabular}{|c|c|c|c|c|}
\hline \multicolumn{5}{|c|}{ Radiotherapy after surgery } \\
\hline No & 1.00 & \multicolumn{3}{|c|}{ Reference } \\
\hline Yes & 2.12 & 1.48 & 3.03 & $<0.001$ \\
\hline \multicolumn{5}{|c|}{ Chemotherapy after surgery } \\
\hline No & 1.00 & \multicolumn{3}{|c|}{ Reference } \\
\hline Yes & 1.69 & 1.17 & 2.44 & 0.005 \\
\hline \multicolumn{5}{|c|}{ Multivariate analysis } \\
\hline \multicolumn{5}{|l|}{ rs13042395 } \\
\hline $\mathrm{CC}+\mathrm{CT}$ & 1.00 & \multicolumn{3}{|c|}{ Reference } \\
\hline$\pi$ & 0.62 & 0.38 & 0.99 & 0.046 \\
\hline \multicolumn{5}{|c|}{ Depth of tumor invasion } \\
\hline Tis $+\mathrm{T} 1$ & 1.00 & \multicolumn{3}{|c|}{ Reference } \\
\hline $\mathrm{T} 2$ & 4.23 & 0.94 & 19.11 & 0.06 \\
\hline T3 & 6.37 & 1.54 & 26.40 & 0.01 \\
\hline T4 & 1.47 & 0.13 & 16.94 & 0.76 \\
\hline$P_{\text {trend }}$ & & & & 0.001 \\
\hline \multicolumn{5}{|c|}{ Regional lymph node metastasis } \\
\hline NO & 1.00 & \multicolumn{3}{|c|}{ Reference } \\
\hline N1 & 2.06 & 1.36 & 3.13 & $<0.001$ \\
\hline N2 & 2.88 & 1.70 & 4.86 & 0.001 \\
\hline N3 & 2.08 & 1.01 & 4.30 & $<0.001$ \\
\hline$P_{\text {trend }}$ & & & & 0.047 \\
\hline
\end{tabular}
associated with relapse-free survival for ESCC patients

$\left(\mathrm{N}_{\mathrm{ESCC}}=288\right)$ (Continued)

Radiotherapy after surgery

\begin{tabular}{lclll} 
No & 1.00 & Reference & & \\
Yes & 1.68 & 1.16 & 2.43 & 0.006 \\
\hline $\begin{array}{l}\text { All associations are significant at } P<0.05 \\
\text { ESCC esophageal squamous cell carcinomas, } \\
\text { confidence interval }\end{array}$
\end{tabular}

levels of riboflavin intake via SLC52A3 overexpression promotes tumorigenesis by sustaining cell proliferation and protecting against cell death [14]. Taking this one step further, there might exist a self-balancing regulation system in which $S L C 52 A 3$ could suppress itself when overexpressed in humans. As a result, dysfunction of the SLC52A3 gene could occur, and the ability of RFT2 to transport dietary riboflavin becomes inhibited. This may partly explain why blood riboflavin is deficient, even though the dietary riboflavin was sufficient.

Regarding rs3746803, we found rs3746803 had no association with susceptibility, tumor characteristics and survival of ESCC patients, consistent with a previous study [15]. This could be because the MAF of rs3746803 is too low in the Chaoshan population.

There are some limitations in the present study. First, our study is a retrospective study, which may lead to statistical bias in the analyses. Replicating studies with 
A

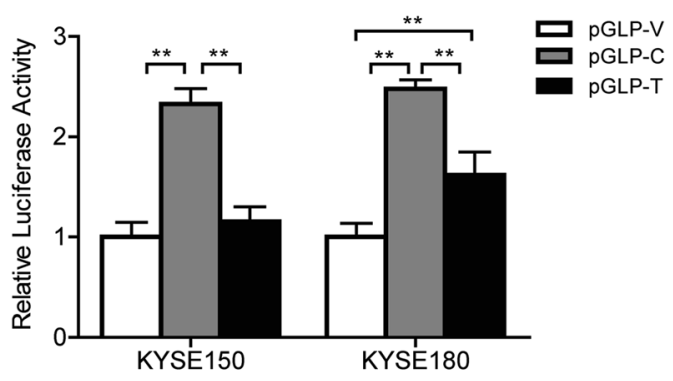

B
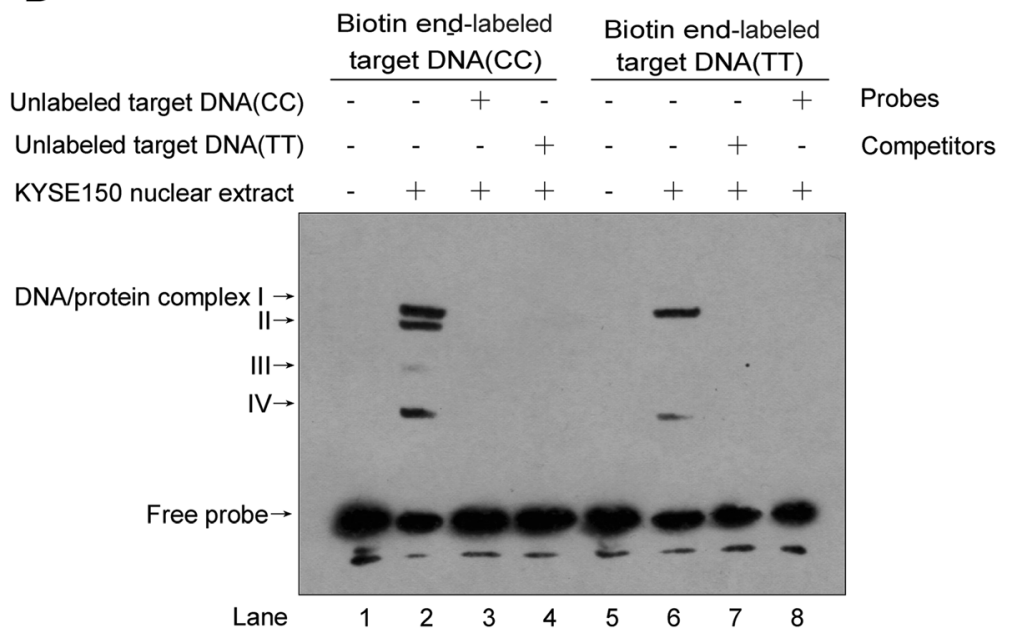

Fig. 2 Promoter activity of rs 13042395 polymorphisms in the SLC52A3 gene in ESCC cells. a Luciferase reporter activity of the SLC52A3 rs 13042395 locus in KYSE150 and KYSE180 cells. Constructions of PGLP-V, PGLP-C or PGLP-T were co-transfected into the cells with pRL-TK in the indicated amounts. The empty vector pGLP-V was used as a control. Firefly luciferase activity was normalized to Renilla luciferase activity of the internal control. The experiments were repeated three times. Error bars indicate $95 \%$ confidence intervals, one asterisk indicates statistical significance with $P<0.05$, two asterisks indicate statistical significance with $P<0.01$. Statistical significance was determined by two-sided one-way analysis of variance along with the Bonferroni post hoc test. $\mathbf{b}$ Electrophoretic mobility shift assay analysis of specific interaction between nuclear proteins and the rs 13042395 site of SLC52A3. The nuclear protein was extracted from KYSE150 cells. Three micrograms of extract were incubated with a biotin end-labeled oligonucleotide GGCCAGTGCACCGTCATTGTGTGGGCTGGG (CC probes, lanes 1 through 4) or GGCCAGTGCACCGTTATTGTGGGGCTGGG (TT probes, lanes 5 through 8) in the rs 13042395 site. Binding specificity was confirmed by chasing labeled CC or TT probes with a 200-fold molar excess of unlabeled CC (lane 4) or $\Pi$ probe (lane 7). Labeled probes free of nuclear extracts migrated as shown in lanes1 and 4. Four shift bands presented in lane 2 (CC polymorphism), but only two in lane 6 (TT polymorphism)

larger sample size and with a prospective design is necessary to clarify the association between rs13042395 and rs3746803 and ESCC. Second, our study indicates that the CC genotype of rs 13042395 promotes SLC52A3 expression, probably via binding with specific transcription factors that generated complexes II and III. However, the identity of the transcription factors, their underlying biological functions, and the mechanisms by which they regulate SLC52A3 expression all remain unknown. All of these scientific issues require further research.

\section{Conclusions}

The present study demonstrates that rs13042395 polymorphisms in the $S L C 52 A 3$ gene play an important role in regional lymph node metastasis and relapse-free survival of ESCC patients. The rs13042395 could enhance
SLC52A3 expression in humans, and this enhancement is probably due to additional transcription factor binding. These results might provide clues for health care planning and clinical research of ESCC, increasing important areas with the application of targeted therapies.

\section{Additional files}

Additional file 1: Table S1. Relapse-free survival for 84 ESCC patients who had radiotherapy after surgery. (DOC $34 \mathrm{~kb}$ )

Additional file 2: Figure S1. Kaplan-Meier analysis of relapse-free survival and overall survival for the rs3746803 loci of SLC52A3 in 288 esophageal squamous cell carcinoma cases. (A) The median relapse-free survival time for $G G$ genotype carriers and (GA + AA) genotype carriers were 25 months and 20 months, respectively. The difference of relapse-free survival time between $G G$ genotype carriers and (GA + AA) genotype carriers was not significant $(P=0.46)$. (B) The median overall 
survival time for $\mathrm{GG}$ genotype carriers and (GA + AA) genotype carriers were 28 months and 27 months, respectively. The difference of overall survival time between $\mathrm{GG}$ genotype carriers and (GA + AA) genotype carriers was not significant $(P=0.92)$. (TIF $1240 \mathrm{~kb}$ )

Additional file 3: Table S2. Univariate analyses and multivariate analysis of factors associated with overall survival for ESCC patients $\left(N_{\mathrm{ESCC}}=288\right) .(\mathrm{DOC} 107 \mathrm{~kb})$

\section{Abbreviations}

$\mathrm{Cl}$, confidence interval; EAC, esophageal adenocarcinoma; EC, esophageal cancer; EMSA, electrophoretic mobility shift assay; ESCC, esophageal squamous cell carcinoma; GWAS, genome-wide association studies; $\mathrm{HR}$, hazard ratio; MAF, minor allele frequency; OR, odds ratio; $\mathrm{PCR}$, polymerase chain reaction; $P_{\text {trend }}$ P value for trend; RFT2, human riboflavin transporter 2; SNPs, single nucleotide polymorphisms

\section{Acknowledgements}

The authors thank all participants in the study. We thank Dr. Stanley Li Lin, Department of Pathophysiology, The Key Immunopathology Laboratory of Guangdong Province, Shantou University Medical College, for the assistance in revising the manuscript.

\section{Funding}

This study was supported by the Natural Science Foundation of China-GuangDong Joint Fund (grants U1301227), the Key Laboratory Project for College and University of Guangdong Province (grant KLB11009), and the Department of Education, Guangdong Government under the Top-tier University Development Scheme for Research and Control of Infectious Diseases. The funders had no role in study design, data collection and analysis, decision to publish, or preparation of the manuscript.

\section{Availability of data and materials}

Data and materials are available from the corresponding authors upon request.

\section{Authors' contributions}

EML and LYX designed the study. HZT, ZYW, YHP, YWX and SSL collected the blood samples and clinical data of patients. JYW was responsible for collection of follow-up data for ESCC patients after surgery. HZT and JWJ were responsible for DNA extraction and SNP genotyping assays. LL and WW were responsible for cell culture, dual luciferase reporter assays and electrophoretic mobility shift assays. JJZ was responsible for statistical considerations of the analysis. HZT drafted the article. EML and LYX and $J J Z$ revised the article. All authors read and approved the final manuscript.

\section{Competing interests}

The authors declare that they have no competing interests.

\section{Consent for publication}

Not applicable.

\section{Ethics approval and consent to participate}

This study was approved by the Ethics Committee of Shantou University Medical College. Written informed consents were obtained from all the study participants.

\footnotetext{
Author details

${ }^{1}$ Key Laboratory of Molecular Biology in High Cancer Incidence Coastal Chaoshan Area of Guangdong Higher Education Institutes, Shantou University Medical College, No. 22, Xinling Road, Shantou 515041, China. 2Department of Biochemistry and Molecular Biology, Shantou University Medical College, No. 22, Xinling Road, Shantou 515041, China. ${ }^{3}$ Department of Oncologic Surgery, Shantou Central Hospital, Affiliated Shantou Hospital of Sun Yat-Sen University, Shantou 515041, China. ${ }^{4}$ Department of Clinical Laboratory, Cancer Hospital of Shantou University Medical College, No.7, Raoping Road, Shantou, Guangdong 515041, China. ${ }^{5}$ Department of Preventive Medicine, Shantou University Medical College, No. 22, Xinling Road, Shantou 515041, China. Institute of Oncologic Pathology, Shantou University Medical College, No. 22, Xinling Road, Shantou 515041, China.
}

Received: 12 August 2015 Accepted: 23 June 2016 Published online: 29 July 2016

\section{References}

1. Torre LA, Bray F, Siegel RL, Ferlay J, Lortet-Tieulent J, Jemal A. Global cancer statistics, 2012. CA Cancer J Clin. 2015;65(2):87-108.

2. Chen W, Zheng R, Zhang S, Zhao P, Zeng H, Zou X, et al. Annual report on status of cancer in China, 2010. Chin J Cancer Res. 2014;26(1):48-58.

3. GLOBOCAN 2012: Estimated Cancer Incidence, Mortality and Prevalence Worldwide in 2012. http://globocan.iarc.fr/Pages/fact_sheets_population. aspx (2013). Accessed 15 July 2015.

4. Lagergren J, Mattsson F. Diverging trends in recent population-based survival rates in oesophageal and gastric cancer. PLoS One. 2012;7(7):e41352.

5. Enzinger PC, Mayer RJ. Esophageal cancer. N Engl J Med. 2003:349(23):2241-52

6. Shimada H, Nabeya Y, Okazumi S, Matsubara H, Shiratori T, Gunji Y, et al. Prediction of survival with squamous cell carcinoma antigen in patients with resectable esophageal squamous cell carcinoma. Surgery. 2003;133(5):486-94.

7. Abnet CC, Freedman ND, Hu N, Wang Z, Yu K, Shu XO, et al. A shared susceptibility locus in PLCE1 at 10q23 for gastric adenocarcinoma and esophageal squamous cell carcinoma. Nat Genet. 2010;42(9):764-7.

8. Wang LD, Zhou FY, Li XM, Sun LD, Song X, Jin Y, et al. Genome-wide association study of esophageal squamous cell carcinoma in Chinese subjects identifies susceptibility loci at PLCE1 and C20orf54. Nat Genet. 2010:42(9):759-63.

9. Wu C, Hu Z, He Z, Jia W, Wang F, Zhou Y, et al. Genome-wide association study identifies three new susceptibility loci for esophageal squamous-cell carcinoma in Chinese populations. Nat Genet. 2011;43(7):679-84.

10. Abnet CC, Wang Z, Song X, Hu N, Zhou FY, Freedman ND, et al. Genotypic variants at 2 q33 and risk of esophageal squamous cell carcinoma in China: a meta-analysis of genome-wide association studies. Hum Mol Genet. 2012;21(9):2132-41.

11. Wu C, Wang Z, Song X, Feng XS, Abnet CC, He J, et al. Joint analysis of three genome-wide association studies of esophageal squamous cell carcinoma in Chinese populations. Nat Genet. 2014;46(9):1001-6.

12. Yamamoto S, Inoue K, Ohta KY, Fukatsu R, Maeda JY, Yoshida Y, et al. Identification and functional characterization of rat riboflavin transporter 2. J Biochem. 2009;145(4):437-43.

13. Fujimura M, Yamamoto $S$, Murata T, Yasujima T, Inoue $K$, Ohta KY, et al. Functional characteristics of the human ortholog of riboflavin transporter 2 and riboflavin-responsive expression of its rat ortholog in the small intestine indicate its involvement in riboflavin absorption. J Nutr. 2010;140(10):1722-7.

14. Jiang $X R$, Yu XY, Fan JH, Guo L, Zhu C, Jiang $W$, et al. RFT2 is overexpressed in esophageal squamous cell carcinoma and promotes tumorigenesis by sustaining cell proliferation and protecting against cell death. Cancer Lett. 2014;353(1):78-86

15. Ji A, Wang J, Yang J, Wei Z, Lian C, Ma L, et al. Functional SNPs in human C20orf54 gene influence susceptibility to esophageal squamous cell carcinoma. Asian Pac J Cancer Prev. 2011;12(12):3207-12.

16. Ji A, Ma L, Yang J, Xu Y, Wang J, Lian C, et al. The functional SNP rs3746804 in C20orf54 modifies susceptibility to esophageal squamous cell carcinoma. Oncol Res Treat. 2014;37(11):654-7.

17. Gu H, Ding G, Zhang W, Liu C, Chen Y, Chen S, et al. Replication study of PLCE1 and C20orf54 polymorphism and risk of esophageal cancer in a Chinese population. Mol Biol Rep. 2012;39(9):9105-11.

18. Palmer AJ, Lochhead P, Hold GL, Rabkin CS, Chow WH, Lissowska J, et al. Genetic variation in C20orf54, PLCE1 and MUC1 and the risk of upper gastrointestinal cancers in Caucasian populations. Eur J Cancer Prev. 2012;21(6):541-4.

19. Dura P, Bregitha $\mathrm{CV}$, te Morsche RH, Roelofs HM, Kristinsson JO, Wobbes T, et al. GWAS-uncovered SNPs in PLCE1 and RFT2 genes are not implicated in Dutch esophageal adenocarcinoma and squamous cell carcinoma etiology. Eur J Cancer Prev. 2013;22(5):417-9.

20. Piao JM, Shin MH, Kim HN, Song HR, Kweon SS, Choi JS, et al. Replication of results of genome-wide association studies on esophageal squamous cell carcinoma susceptibility loci in a Korean population. Dis Esophagus. 2014:27(8):798-801.

21. Shi YY, He L. SHEsis, a powerful software platform for analyses of linkage disequilibrium, haplotype construction, and genetic association at polymorphism loci. Cell Res. 2005;15(2):97-8. 
22. Xu Y, Chen Q, Yu X, Zhou X, Zheng X, Mao W. Factors influencing the risk of recurrence in patients with esophageal carcinoma treated with surgery: A single institution analysis consisting of 1002 cases. Oncol Lett. 2013;5(1):185-90.

23. Lorenz D, Origer J, Pauthner M, Graupe F, Fisseler-Eckhoff A, Stolte M, et al. Prognostic risk factors of early esophageal adenocarcinomas. Ann Surg. 2014;259(3):469-76.

24. Yang CS, Miao J, Yang W, Huang M, Wang T, Xue H, et al. Diet and vitamin nutrition of the high esophageal cancer risk population in Linxian. China Nutr Cancer. 1982;4(2):154-64.

25. van Rensburg SJ, Benade AS, Rose EF, du Plessis JP. Nutritional status of African populations predisposed to esophageal cancer. Nutr Cancer. 1983;4(3):206-16.

26. Guo WD, Li JY, Blot WJ, Hsing AW, Chen JS, Fraumeni Jr JF. Correlations of dietary intake and blood nutrient levels with esophageal cancer mortality in China. Nutr Cancer. 1990;13(3):121-7.

27. Siassi F, Ghadirian P. Riboflavin deficiency and esophageal cancer: a case control-household study in the Caspian Littoral of Iran. Cancer Detect Prev. 2005:29(5):464-9.

28. Webster RP, Gawde MD, Bhattacharya RK. Modulation of carcinogen-induced DNA damage and repair enzyme activity by dietary riboflavin. Cancer Lett. 1996;98(2):129-35.

29. Blot WJ, Li JY, Taylor PR, Guo W, Dawsey S, Wang GQ, et al. Nutrition intervention trials in Linxian, China: supplementation with specific vitamin/mineral combinations, cancer incidence, and disease-specific mortality in the general population. J Natl Cancer Inst. 1993;85(18):1483-92.

30. Dawsey SM, Wang GQ, Taylor PR, Li JY, Blot WJ, Li B, et al. Effects of vitamin/mineral supplementation on the prevalence of histological dysplasia and early cancer of the esophagus and stomach: results from the Dysplasia Trial in Linxian, China. Cancer Epidemiol Biomarkers Prev. 1994;3(2):167-72.

31. Wang GQ, Dawsey SM, Li JY, Taylor PR, Li B, Blot WJ, et al. Effects of vitamin/mineral supplementation on the prevalence of histological dysplasia and early cancer of the esophagus and stomach: results from the General Population Trial in Linxian, China. Cancer Epidemiol Biomarkers Prev. 1994;3(2):161-6.

32. He Y, Ye L, Shan B, Song G, Meng F, Wang S. Effect of riboflavin-fortified salt nutrition intervention on esophageal squamous cell carcinoma in a high incidence area, China. Asian Pac J Cancer Prev. 2009;10(4):619-22.

\section{Submit your next manuscript to BioMed Central and we will help you at every step:}

- We accept pre-submission inquiries

- Our selector tool helps you to find the most relevant journal

- We provide round the clock customer support

- Convenient online submission

- Thorough peer review

- Inclusion in PubMed and all major indexing services

- Maximum visibility for your research

Submit your manuscript at www.biomedcentral.com/submit 